\title{
Can EBSD Patterns Be Used for Determination of Grain Boundary Inclination?
}

\author{
Michael Chapman ${ }^{1}$, Saransh Singh $^{1}$, Marc De Graef $^{1}$ \\ ${ }^{1}$ Dept. of Materials Science and Engineering, Carnegie Mellon Univ., Pittsburgh PA 15213, USA
}

The functional properties of many polycrystalline materials are strongly correlated with the grain boundary character. While electron or optical microscopes can be used to study grain boundaries, they only reveal the 2D nature by means of cross sectional images. To obtain an accurate five-parameter description of grain boundaries, a full 3D characterization is required. While there exist several theoretical and experimental techniques to study the grain structures in 3D, e.g., stereology, serial sectioning using a Focused Ion Beam (FIB), and high energy x-ray diffraction at a synchrotron facility, these methods are either statistical, destructive or not easily accessible.

In this contribution, we describe preliminary results of a novel technique, combining Monte Carlo electron trajectory simulations and dynamical Electron Backscatter Diffraction Pattern (EBSP) simulations to extract the grain boundary inclination from EBSD observations. The Monte Carlo simulations closely follow the model described in [1]. In this model, the material is assumed to be isotropic. It is also assumed that only elastic scattering events change the direction of the electrons, while the inelastic events are responsible for the energy loss. Apart from a few special energy loss mechanisms, e.g., Bremsstrahlung radiation, the electron loses energy in discrete collision events. However, the averaging of all such events along the trajectory of the electron leads to significant simplifications and it is assumed that the electron loses energy at a fixed rate per unit distance along its trajectory. This approximation is known as the Continuous Slowing Down Approximation (CSDA) and is valid for electrons having energies in the multiple keV range. The Rutherford scattering cross section is used to model the elastic scattering process. The dynamical simulation of the EBSPs follows the model described in [2]. The calculated patterns are tuned to the crystallography of the sample (crystal orientation, symmetry) and the microscope parameters (acceleration voltage, sample tilt, detector geometry etc.). The model calculates the depth and energy distribution of the electrons using Monte Carlo simulations and integrates the backscatter probability over the corresponding depth range to determine the intensity at a detector pixel as a function of energy and the pixel location. The backscatter probability is then given as the squared modulus of the electron wavefunction, which is typically computed using either the Bloch wave approach or the scattering matrix approach.

The geometry used for the pattern simulations is shown in Fig. 1(a). The sample is composed of two grains with different crystal orientations. Far from the grain boundary, the observed pattern will be that of a single orientation, i.e., grain A or B. However, as the electron beam is rastered across the grain boundary, the observed pattern will become a superposition (mixture) of pattern contributions from both grains A and B. As a first order approximation, the mixing of the two patterns can be assumed to be linear, with relative weights depending on the number of backscattered electrons originating inside each of the respective grains, normalized by the total number of backscattered electrons. The number of electrons coming from the two grains as a function of the distance of the electron beam to the grain boundary and the grain boundary inclination angle with respect to the surface is shown in Fig. 1(b). Shallow grain boundaries show the largest changes in the number of BSEs. Fig. 2(a) and (b) show simulated EBSPs for two random crystal orientations and Fig. 2(c) shows the expected pattern when the number of BSEs is evenly split between the two grains. We will present preliminary results on 
the feasibility of extracting the grain boundary inclination angle from the mixture EBSD pattern using a dot-product analysis between pure A and B patterns and the mixture pattern; we will show that, at least for shallow inclination angles, the grain boundary inclination can indeed be extracted from the mixture EBSD pattern.

\section{References}

[1] D.C. Joy in "Monte Carlo Modeling for Electron Microscopy and Microanalysis", (1995, Oxford University Press, New York) p. 25

[2] P.G. Callahan and M. De Graef, Microscopy and Microanalysis, 19, 1255-1265 (2013).

[3] Research supported by the Air Force Office of Scientific Research, MURI contract \# FA9550-12-1-0458.
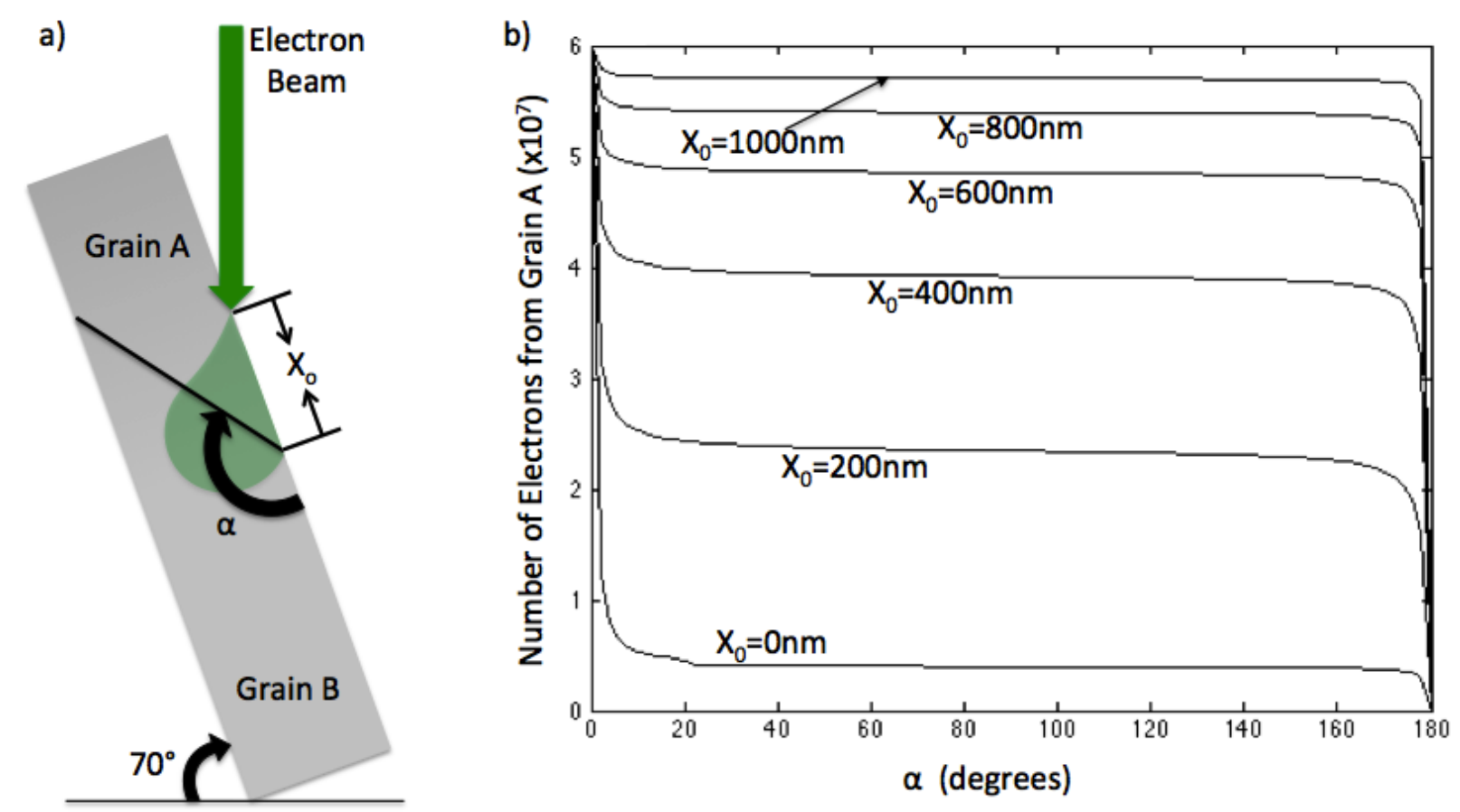

Figure 1. a) Sample geometry with a grain boundary inclination angle $\alpha$ and distance $X_{0}$ between electron beam incidence and boundary; b) number of electrons from Grain A vs. inclination angle $\alpha$ for six different $X_{0}$ values.

a)

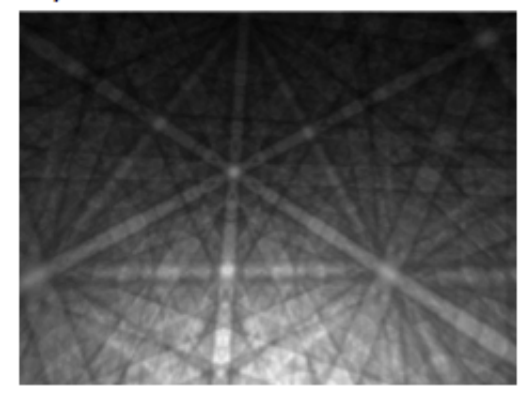

b)

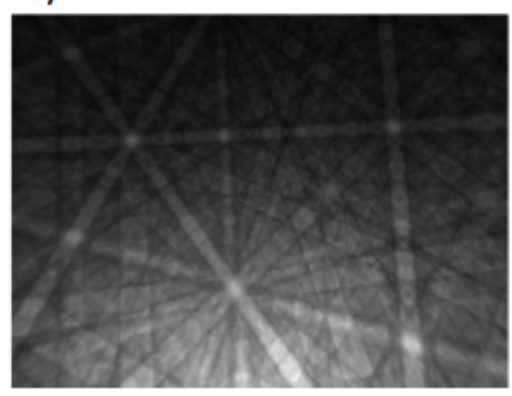

c)

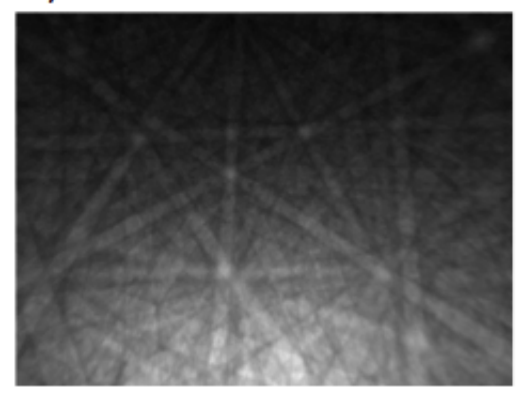

Figure 2. a) EBSP for Grain A, b) EBSP for Grain B, c) EBSP for the grain boundary between Grain $A$ and Grain $B$ where 50 percent of the electrons come from each grain. 